\title{
Influence of pain-related psychological factors on therapeutic outcomes in patients with chronic low back pain after oxygen-ozone treatment: a case-series
}

\author{
Francesca Cantele (1), Lucrezia Tognolo (1,2), Francesca Caneva (1), Emanuela \\ Formaggio (2), Valentina Copetti (1), Andrea Venturin (3), Allegra Caregnato (1), \\ Stefano Masiero $(1,2)$
}

(1) Physical Medicine and Rehabilitation School, University of Padua, Padova, Italy; (2) Department of Neurosciences, Physical Medicine and Rehabilitation Unit, University of Padua, Padova, Italy; (3) Orthopedic Rehabilitation Unit, General Hospital, University of Padua Medical Center, Padova, Italy.

This article is distributed under the terms of the Creative Commons Attribution Noncommercial License (CC BY-NC 4.0) which permits any noncommercial use, distribution, and reproduction in any medium, provided the original author(s) and source are credited.

\begin{abstract}
The aim of the study was to identify the variables related to therapeutic success of intramuscular oxygen-ozone (O2O3) in patients with chronic low back pain (LBP). Twenty-one patients underwent an eight-session intramuscular-paravertebral $\mathrm{O} 2 \mathrm{O} 3$ treatment with a weekly frequency. Numeric Rating Score for pain (NRSp), anxiety (NRSa), mood (NRSm), Oswestry Disability Index (ODI) and Physical and Mental Component Summary scores (PCS and MCS) of Short Form 12 (SF-12) were assessed baseline, after the treatment and at six-months followup. The ODI and NRSp scores showed significant improvement at the end of the treatment: the improvement in pain was maintained also at 6 months' follow-up. A significant correlation between baseline NRSa and the variation of the NRSp and ODI was observed. There was a correlation between NRSm at baseline and the variation of the ODI. A moderate correlation between MCS-12 at baseline and the variation of the NRSp score was found. Surprisingly, patients with a more compromised psychological well-being due to LBP had better results after oxygen-ozone therapy. Therefore, NRSa, NRSm and MCS-12 scores could be useful predictors of good therapeutic outcome.
\end{abstract}

Key Words: Low back pain, oxygen-ozone therapy, musculoskeletal injection therapy, psychological factors, anxiety.

Eur J Transl Myol 31 (3): 9906, 2021 doi: 10.4081/ejtm.2021.9906

Chronic Low Back Pain (LBP) is a common condition among the general population and its management often represents a challenge for the clinicians, due to the heterogeneous etiologies and physiopathology. The social costs of LBP could be considerable and some patients could experience restrictions in their work and family activities, emotional distress and quality of life's impairment. ${ }^{1}$ Furthermore, several studies demonstrated the influence of psychological factors in the development, persistence, and treatment of chronic LBP. ${ }^{2}$ Coping style, depression, anxiety, catastrophizing, and level of pain acceptance are important factors to be considered in the global rehabilitation process of the patients with LBP. ${ }^{3}$ In the last few years, lumbar intramuscular-paravertebral injections of an oxygenozone $\left(\mathrm{O}_{2} \mathrm{O}_{3}\right)$ mixture have been proposed in the chronic LBP's management, alongside the conventional treatments (non-steroidal anti-inflammatory drugs, antidepressants, exercise therapy and psychosocial interventions). ${ }^{4} \mathrm{O}_{2} \mathrm{O}_{3}$ is a gas mixture of medical oxygen and ozone which is produced from pure oxygen passing through a high voltage gradient in a medical generator. ${ }^{5}$ Its effectiveness on LBP has been ascribed to the modulation of the arachidonic acid metabolism, that could explain the anti-inflammatory effect, and to the promotion of the local micro-vascularization, reducing the ischemia and the inflammatory edema in the periradicular area. ${ }^{6,7}$ Moreover, the Reactive Oxygen Species (ROS) generated by the interaction between the $\mathrm{O}_{2} \mathrm{O}_{3}$ molecules and the biological tissues may lead to the descendent antinociceptive system's activation, generating a direct effect against the pain. ${ }^{8}$ Some trials have shown that $\mathrm{O}_{2} \mathrm{O}_{3}$ treatment is effective in reducing LBP, in improving LBP-related disability and in 
decreasing the intake of analgesics. ${ }^{9-11}$ Apuzzo et al. compared the effects of intramuscular $\mathrm{O}_{2} \mathrm{O}_{3}$ infiltration and Global Postural Re-education (GPR) on LBP's severity, finding a major reduction in pain levels among the $\mathrm{O}_{2} \mathrm{O}_{3}$ patients than in GPR-alone patients. ${ }^{5}$ Recently, De Oliveira Magalhaes et al. performed a review to investigate the therapeutic results of percutaneous injection of $\mathrm{O}_{2} \mathrm{O}_{3}$ for LBP and affirmed that additional studies are needed before the role of $\mathrm{O}_{2} \mathrm{O}_{3}$ can be established in the management of LBP. ${ }^{12}$ To the best of the authors' knowledge, none of the studies has so far evaluated the patient's physical and mental characteristics, which could influence the outcome of the $\mathrm{O}_{2} \mathrm{O}_{3}$ intramuscular-paravertebral treatment. Some authors have proposed a pre-intervention psychological screening in lumbar surgery patients to identify suitable candidates and to predict possible complications or poor outcome from treatment. ${ }^{13}$ Similarly, we believe it is important to identify those patients who should benefit more from the intervention with $\mathrm{O}_{2} \mathrm{O}_{3}$, because it would result in an optimization of the therapeutic chances and in a better public-health-care resources management. Secondly, we wanted to confirm the previous literature's results about the effectiveness of $\mathrm{O}_{2} \mathrm{O}_{3}$ therapy in the improvement of LBP. Therefore, we designed this retrospective observational study to define which patient's physical and psychological variables are more related to the success of the treatment.

\section{Materials and Methods}

This retrospective observational study was conducted between February 2019 and September 2020 in the Orthopedic and Rehabilitation outpatient Department of Padua University Hospital (protocol number 70254). Written informed consent was obtained from all the patients. Inclusion criteria were the following: age $>18$ years; moderate or severe chronic LBP $(\geq 3$ on a $10-\mathrm{cm}$ pain Numeric Rating Scale [NRSp]) of a mechanical and degenerative nature, even if not continuous, experienced for at least 3 months non-responsive to conservative treatment; diagnosis of discopathy, articular facet syndrome supported by radiographs or Magnetic Resonance Imaging (MRI) investigation with evidence of spondylodiscoarthrosis, narrowing of intersomatic spaces. Exclusion criteria were: skin infection or changes at the injection site; favism (glucose-6-phosphate dehydrogenase deficiency); severe spine deformities on coronal and/or sagittal plane; neoplasia; heart failure; pregnancy; equine cauda syndrome, progressive neurologic deficit; previous spine surgery; diabetic neuropathy. All patients were treated using the same medical ozone generator. With the patient lying in a prone position, bilateral paraspinal intramuscular $\mathrm{O}_{2} \mathrm{O}_{3}$ injections (10 $\mathrm{mL}$ for each side) at the affected level with a $\mathrm{O}_{3}$ concentration of $20 \mu \mathrm{g} / \mathrm{mL}$ using a $22 \mathrm{G} 0.7$ x $40 \mathrm{~mm}$ needle were performed. Before injection, the locus dolendi has been confirmed through the segmental semeiotic with an axial and lateral pressure of the lumbar vertebrae. Before the procedure, skin disinfection was carried out with sterile gauze and chlorhexidine. The procedure was performed by a well-instructed physician. All patients underwent an eight-session intramuscular paravertebral $\mathrm{O}_{2} \mathrm{O}_{3}$ treatment. The injections were performed with weekly frequency, for a total duration of eight weeks.

In accordance with normal clinical practice all the patients were measured on pain and disability scales. Local pain was assessed by pain Numeric Rating Scale (NRSp, from 0-10 where 0 is "not noticeable at all” and 10 is "the worst pain imaginable"); anxiety and mood were evaluated by the anxiety Numeric Rating Scale (NRSa, from 0-10 where 0 is "not noticeable at all" and 10 is "the highest anxiety level") and mood Numeric Rating Scale (NRSm, from $0-10$ where 0 is "a total depressed mood" and 10 is "mood not influenced by pain”). The validated Italian version of the Oswestry Disability Index (ODI), a self-completed questionnaire which consists in 10 sections concerning intensity of pain, lifting, ability to care for oneself, ability to walk, ability to sit, sexual function, ability to stand, social life, sleep quality, and ability to travel, has been used for the analysis of everyday life activities and disability.

The Short Form-12 (SF-12) score was used for the analysis of physical and mental health. It consisted in 12 items divided in 8 domains. The SF-12 has two summary measures: the Physical and Mental Component Summary scores (PCS and MCS) with higher scores representing better health status. ${ }^{14}$

Each outcome measure was recorded prior to the procedure (T0) and after the last session (T1). The follow up was performed 6 months after the last injection (T2): due to the restrictions for SARS-CoV-2 pandemics, we were not able to visit patients at this time. Therefore, patients were contacted by telephone and gave their consent to participate. Their answers about NRSp, NRSa and NRSm were immediately transcribed. We decided not recording the ODI and SF-12 because of the difficulty in administering these questionnaires by telephone interview. Descriptive statistics (mean and standard deviations) were utilized to describe the distribution of the results with respect to statistical quantitative features. A paired sample two-tailed t-test $(p<0.05)$ was performed to obtain statistical inference regarding time differences (T0 vs. T1) in clinical scores. Analysis of Variance (ANOVA) for repeated measures was applied to NRSp, NRSa and NRSm scores with the factor "time" (T0, T1, and T2). A post hoc two-tailed t-test was used whenever appropriate. Finally, the Pearson correlation coefficient was used to assess the relationships between the NRSa, NRSm, MCS-12 scores and the differences (T1-T0) of the NRSp, ODI, PCS-12 scores. P $<005$ was considered statistically significant. Software statistics IBM SPSS (25.0) (IBM, Armonk, NY, USA) was used. Correlations were defined as strong $>0.60$, moderate $0.30-0.60$ and weak $<0.30$, respectively. ${ }^{15}$ 
Table 1. Mean scores of Numeric Rating Score for pain (NRSp), anxiety (NRSa), mood (NRSm), Oswestry Disability Index (ODI \%), Physical and Mental Component Summary scores (PCS-12 and MCS-12) at T0 (before the treatment), at T1 (after the treatment) and $\triangle(T 1-T 0)$ (mean and standard deviation).

\begin{tabular}{ccccc}
\hline & T0 & T1 & $\Delta(\mathbf{T 1}-\mathbf{T 0})$ & \multicolumn{2}{c}{ p-value } \\
\hline NRSp & $6.59 \pm 1.75$ & $5.06 \pm 2.76$ & $-1.53 \pm 3.06$ & 0.03 \\
\hline NRSa & $3.81 \pm 2.79$ & $3.69 \pm 2.95$ & $-0.12 \pm 4.43$ & 0.91 \\
\hline NRSm & $5.38 \pm 2.21$ & $5.81 \pm 2.23$ & $0.44 \pm 3.20$ & 0.56 \\
\hline ODI index (\%) & $33 \pm 14.25$ & $26 \pm 14.32$ & $-8 \pm 12.28$ & 0.01 \\
\hline PCS-12 & & & & 0.00 \\
\hline MCS-12 & $33.99 \pm 7.89$ & $39.10 \pm 10.69$ & $5.43 \pm 6.65$ & 0.74 \\
\hline
\end{tabular}

\section{Results}

\section{Demography}

31 patients met the eligibility criteria. 4 patients declined the treatment after reading informed consent and 2 after the first session, because they were afraid of the needle. 4 patients referred to be in trouble within reaching the hospital because of the sanitary emergency COVID-19 related and were excluded. The study sample was finally formed by 21 patients (10 females, 11 males, age 27-81 years). In 9 patients the pain was present by 12 months or less and in 11 patients by more than 12 months. 10 patients received the treatment in the spring-summer period (May-September), 11 patients were treated in the autumn-winter period (October-April).

\section{Outcome measures}

Table 1 shows the results of outcome measures. A significant decrease was observed for NRSp and for ODI scores after the treatment (T1) (respectively, $\mathrm{p}=0.03$ and $\mathrm{p}=0.01$ ), whereas a significant increase was detected for PCS-12 ( $<<0.01)$. Six months after the end of the treatment (T2), the patients were asked to give a score about their level of NRSp, NRSa and NRSm by that time. ANOVA disclosed a significant main effect for the factor "time" ( $p<0.005)$. A positive trend in terms of parameters' improvement was confirmed (Figure 1). The differences between NRSp at T0 and T1 $(\mathrm{p}<0.01)$ and at T0 and T2 (p <0.005) were statistically significant.
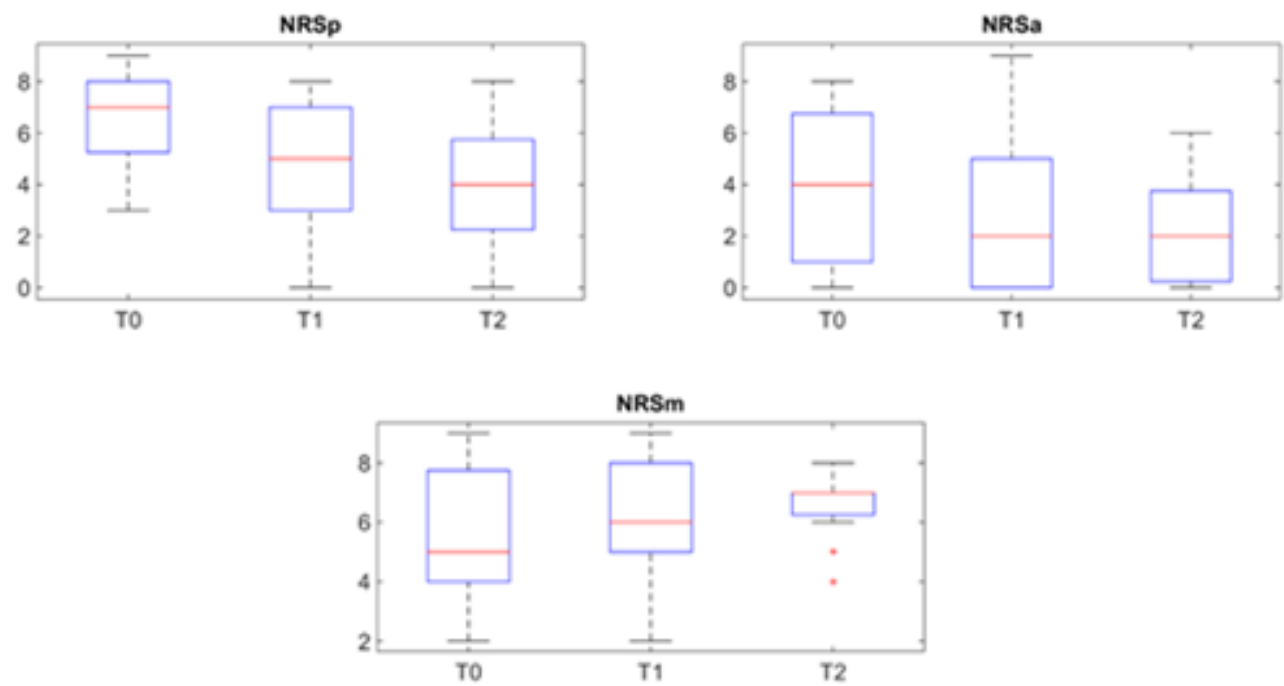

Fig 1. NRSp, NRSa and NRSm scores at T0,T1 and T2. On each box, the central red line indicates the median, and the bottom and top edges of the box indicate the 25th and 75th percentiles, respectively. The whiskers extend to the most extreme data points not considered outliers, and the outliers are plotted individually using the '+' symbol. 


\section{Outcomes in patients with chronic low back pain after oxygen-ozone treatment}

Eur J Transl Myol 31 (3): 9906, 2021 doi: 10.4081/ejtm.2021.9906

\section{Correlations}

A significant moderate correlation between NRSa at T0 and the variation T1-T0 of the NRSp score ( $r=-0.48, \mathrm{p}$ $=0.03$ ) was observed (Figure 2a). Moreover, there was a significant moderate correlation between NRSa at T0 and the variation of the ODI index $(r=-0.55, \mathrm{p}=0.015)$ (Figure 2b). Patients with higher levels of pain-related anxiety at the baseline reported better results in terms of pain reduction and disability improvement at the end of the treatment. There was no statistically significant association between NRSa at T0 and the variation of the PCS-12 score $(r=-0.05, p=0.861)$.

There was a significant strong correlation between NRSm at T0 and the variation of the ODI index ( $r=0.60$, $\mathrm{p}=0.009$ ) (Figure 2c). Patients with more pain-related depressed mood at the baseline show better results in terms of disability reduction at the end of the treatment. There was no statistically significant association between NRSm at T0 and the variation of the NRSp score ( $\mathrm{r}=$ $0.05, \mathrm{p}=0.84$ ) or between NRSm at T0 and the variation of the PCS-12 score $(r=-0.14, p=0.613)$.

The analysis revealed a significant moderate correlation between MCS-12 at T0 and the variation of the NRSp score $(r=0.51, p=0.022)$ (Figure $2 d)$. The patients who reported lower scores in mental health perception index before the treatment, had a greater reduction in pain score. There was no significant association between MCS-12 at T0 and the variation of the PCS-12 score ( $\mathrm{r}=$ $-0.14, p=0.613$ ), while the correlation between MCS-12 at $\mathrm{T} 0$ and the variation of the ODI index was found moderate and closed to significance $(r=0.45, p=0.055)$ (Figure 2e).
Analysis of other variables' influence on treatment outcome

The following variables were then analyzed: age, sex, pain duration and treatment season. There were not statistically differences in the variation of NRSp score, ODI index or PCS-12 scores between males and females, at the end of the treatment, compared to the same measures at baseline. In the same way, there were not differences in the variation of NRSp score, ODI index or PCS-12 scores between $<50$ years old and $>50$ years old patients. Pain duration or the seasonality of the treatment didn't influence the variation of NRSp score, ODI index or PCS-12 scores.

\section{Discussion}

Our work is the first that evaluate which patient's physical and mental characteristics may influence the outcome of the $\mathrm{O}_{2} \mathrm{O}_{3}$ intramuscular-paravertebral treatment. Surprisingly, in our study, patients with higher levels of pain-related anxiety at the baseline reported better results in terms of pain reduction and disability improvement at the end of the treatment. Similarly, the patients with more pain-related depressed mood showed better results in terms of disability reduction. The patients reporting lower scores in MCS-12 before the treatment, and who consequently complained of higher psychological impact of the painful situation on daily life, had a greater reduction of pain and disability. By contrast, some previous studies reported that cognitive and psychological factors, such as pre-surgical pain somatization, depression and anxiety are predictors of poor response to spinal surgery and to conventional treatment in patients with LBP. ${ }^{13,16}$
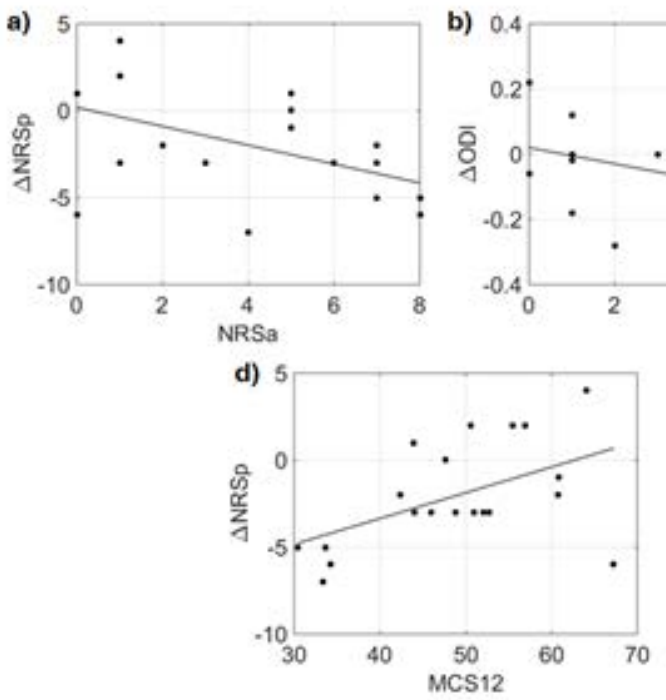
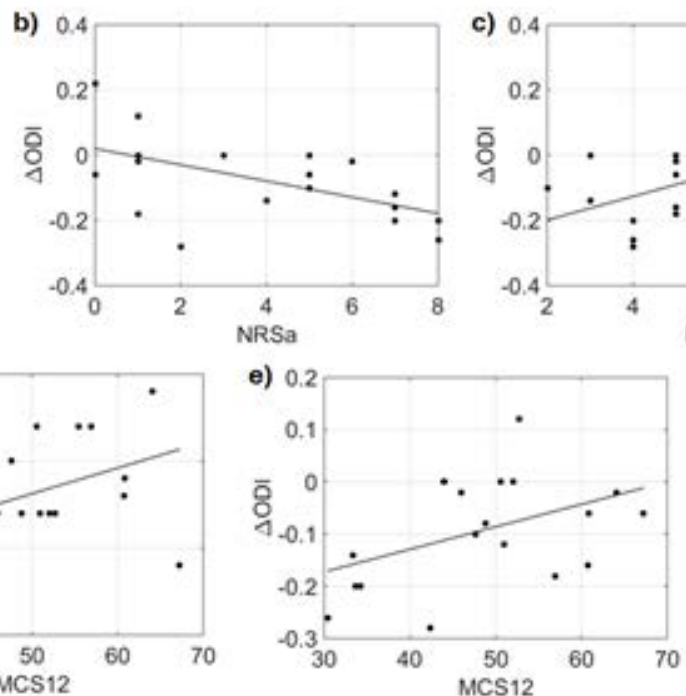

Fig 2. Pearson correlation between NRSa at TO and the differences (T1-T0) of NRSp ( $\triangle N R S p)$ (correlation coefficient $r=-0.48, p=0.03)(a)$; between NRSa at T0 and $\triangle O D I(r=-0.55, p=0.015)(b)$; between NRSm at T0 and $\triangle N R S p(r=0.6, p=0.009)(c)$; between MCS-12 and $\triangle N R S p(r=0.51, p=0.022)(d)$; between MCS-12 and $\triangle O D I(r=0.45, p=0.055)(e)$. 
Nevertheless, these studies considered as predictors some merely psychiatric indexes, which reflect the subject's global psychological status. This sphere may be affected by other aspects as well as the LBP. Our study, indeed, considered those psychological variables strictly related to the pain as a symptom. In fact, NRSa and NRSm represent respectively the level of pain-related anxiety and pain-related mood. The MCS-12 score of SF-12 considers the psychological impact of the painful situation on daily life. Some principles of neuroscience about the central pain modulation pathway could support our findings. Central Sensitization (CS) is meant as the set of the processes that contributed to alter the perception of the painful stimuli at the level of the SNC and its role in pain perception is accentuated in patients with chronic LBP, which exhibit a lower tolerance for pain, if compared with healthy people. ${ }^{17,18}$ There is strong evidence that maladaptive psychological factors (anxiety, depression, catastrophic tendencies, beliefs about the disease and somatization) may be CS predictors in LBP (cognitive-emotional sensitization process) and individuals with high anxious personality type are more susceptible to show CS symptoms or higher scores in CS assessments. ${ }^{19,20}$ It is safe to assume that individuals with higher levels of pain-related anxiety or pain-related depressed mood and more compromised mental health perception are more subject to a CS process within the group with LBP. However, this is just an assumption, since, to the authors knowledge, no study has so far investigated the association between NRSa or NRSm score and levels of CS. The $\mathrm{O}_{2} \mathrm{O}_{3}$ therapy has shown to exert an indirect effect in promoting the descending antinociceptive system, which is particularly involved in the process of CS. Moreover, some papers have shown the effectiveness of $\mathrm{O}_{2} \mathrm{O}_{3}$ therapy in patients affected by disorders with high CS levels, underlining the potential role of the $\mathrm{O}_{2} \mathrm{O}_{3}$ therapy in contrasting the $\mathrm{CS}$ process. ${ }^{21,22}$ It could be hypothesized that, in patients with higher levels of pain-related anxiety and pain-related depressed mood, the $\mathrm{O}_{2} \mathrm{O}_{3}$ therapy may play a role also in the remodulation of the perception of the pain and on the kinesiophobic extent, with a consequent improvement in pain and disability, much more than in patients with low central pain component. A second explanation of our results may be found in the role of the serotonergic system. It is known that a deficit in the serotonergic pathway is related to mood and anxiety disorders. ${ }^{23}$ Serotonin is a key neuromodulator in the transmission of pain in the descending nociceptive modulatory pathways. ${ }^{24}$ Persons with lower levels of serotonin may show higher levels of $\mathrm{CS}^{25}$ Some studies have found that people with chronic LBP show lower serum serotonin levels when compared to healthy persons. ${ }^{26}$ Other authors reported a moderate increase of serotonin levels after autohemotherapy with ozone in patients with fibromyalgia, a disorder linked to CS, such as chronic LBP: this was matched to an improvement in symptomatology too. ${ }^{27}$ Thus, we think that persons with a deficit of serotonin hormone and with consequently higher levels of pain-related anxiety and higher levels of CS could probably be favored by the $\mathrm{O}_{2} \mathrm{O}_{3}$ therapy, also trough the increase of $\mathrm{O}_{2} \mathrm{O}_{3}$-induced serotonin levels. This may result in the clinical evidence of pain reduction and disability improvement, even if there is a lack of studies which correlates the variation of serum serotonin values and the improvement of pain levels in LBP. The mistrust of a placebo effect or a needle effect of the $\mathrm{O}_{2} \mathrm{O}_{3}$ therapy in more psychologically compromised patients must be dispelled by the finding of Paoloni et al. (2009), that comparing intramuscular injections of $\mathrm{O}_{2} \mathrm{O}_{3}$ with simulated lumbar paravertebral injections, that shows good results only in those patients who received a true treatment. ${ }^{9}$ For what concern the other variables analyzed, in our study age, sex, pain length or season of treatment did not seem to affect the therapeutic outcome. If confirmed, there would be some significant therapeutic implications, especially for older patients, who could benefit the same as young people from a mini-invasive and limited side-effects therapeutic intervention. Furthermore, about season of treatment, other authors reported that weather conditions may influence subjective reporting of low back pain significantly and they should be considered in clinical treatment of the patient with chronic LBP. ${ }^{28}$ As regards of secondary endpoint, we observed a significative reduction in pain level at the end of the treatment, compared to the baseline score (T0 vs T1): this result agrees with the previous literature. In their work, Apuzzo et al. (2014) reported an approximately 6-point reduction in VAS (Visual Analog Scale) score at the end of the treatment, but the initial pain levels were higher, compared to our sample. ${ }^{5}$ We also investigated the pain score six months after the treatment. The reduction in pain was confirmed and this supports the hypothesis of a long-term effect of the $\mathrm{O}_{2} \mathrm{O}_{3}$ therapy on the pain levels' reduction. Moreover, our patients showed a significant improvement in the perceived functional status and disability, according to the ODI. This result confirms the work of Biazzo et al. who found an average reduction of $9 \%$ in the ODI scores after the intramuscular paravertebral $\mathrm{O}_{2} \mathrm{O}_{3}$ injections in patients with chronic LBP. ${ }^{10}$ We also reported an improvement in the global physical health perception after the treatment.

On the other hand, our study has some limitations. Firstly, the follow up at six months could be performed only in a remote way and only for few parameters, because of the sanitary emergency COVID-19-related. Secondly, the small number of patients in our sample was due to the fact that, according to the guidelines, we prescribed $\mathrm{O}_{2} \mathrm{O}_{3}$ therapy only for those patients who have not benefited from conventional non-invasive therapies and which are a minority in the number of patients with LBP. Further studies are needed in order to confirm our results. In particular it would be interesting to investigate whether the predictive variables evidenced in our study will prove to be valid and useful also in other ways of 
$\mathrm{O}_{2} \mathrm{O}_{3}$ administration, e.g. in the peridural way or in the autohemotransfusion.

In conclusion, our surprising results show that people who reported more compromised psychological wellbeing due to LBP had better outcomes after $\mathrm{O}_{2} \mathrm{O}_{3}$ treatment. Therefore, baseline NRSa, NRSm and MCS12 scores could be useful indicators of good therapeutic outcome in $\mathrm{O}_{2} \mathrm{O}_{3}$ intramuscular paravertebral therapy in chronic LBP. This evidence identify those patients who should benefit more from this innovative therapy in terms of pain and disability reduction.

Second, we confirmed previous literature on effectiveness of intramuscular paravertebral injections of $\mathrm{O}_{2} \mathrm{O}_{3}$ in reducing pain as well as disability in patients suffering with chronic LBP and that ozone injections are successful in improving global physical health perception.

\section{List of acronyms}

aNRS - anxiety Numeric Rating Scale

CS - Central Sensitization

GP - Global Postural Re-education

LBP - Low Back Pain

MCS - Mental Component Summary

mNRS - mood Numeric Rating Scale

MRI - Magnetic Resonance Imaging

O2O3 - Oxygen-Ozone

ODI - Oswestry Disability Index

PCS - Physical Component Summary

pNRS - pain Numeric Rating Scale

ROS - Reactive Oxygen Species

SF-12 - Short Form-12

\section{Authors contributions}

FC conceptualization, data curation, investigation, writing of original draft; LT conceptualization, methodology, writing of original draft; FC investigation; EF formal analysis; VC Conceptualization and data curation; AV investigation; AC data curation; SM writing - review and editing; supervision.

\section{Acknowledgments}

None.

\section{Funding}

This research received no external funding.

\section{Conflict of Interest}

All Authors declare no conflict of interest

\section{Ethical Publication Statement}

We confirm that we have read the Journal's position on issues involved in ethical publication and affirm that this report is consistent with those guidelines.

\section{Corresponding Author}

Lucrezia Tognolo, via Giustiniani 2, 35128 Padova, Italy ORCID iD: 0000-0002-4239-4859

E-mail: lucrezia.tognolo@unipd.it
E-mails and ORCID iD of co-authors

Francesca Cantele: francyski@hotmail.it ORCID iD: 0000-0001-9906-890X

Francesca Caneva: francesca.caneva10@gmail.com ORCID iD: 000-0002-7625-8097

Emanuela Formaggio: emanuela.formaggio@unipd.it ORCID iD: 0000-0002-3417-0388

Valentina Copetti: val.copetti@gmail.com

ORCID iD: 0000-0002-8080-5313

Andrea Venturin: andrea.venturin@aopd.veneto.it ORCID iD: 0000-0002-5716-1126

Allegra Caregnato: allegracaregnato95@gmail.com ORCID iD: 000-0001-8926-5301

Stefano Masiero: stef.masiero@unipd.it; ORCID iD: 0000-0002-0361-4898

\section{References}

1. Maher C, Underwood M, Buchbinder R. Nonspecific low back pain. Lancet. 2017 Feb 18;389(10070):736-747. doi: 10.1016/S01406736(16)30970-9. Epub 2016 Oct 11.

2. Pincus T, Burton AK, Vogel S, Field AP. A systematic review of psychological factors as predictors of chronicity/disability in prospective cohorts of low back pain. Spine (Phila Pa 1976). 2002 Mar 1;27(5):E109-20. doi: 10.1097/00007632200203010-00017.

3. Turk DC, Fillingim RB, Ohrbach R, Patel KV. Assessment of Psychosocial and Functional Impact of Chronic Pain. J Pain. 2016 Sep;17(9 Suppl):T2149. doi: 10.1016/j.jpain.2016.02.006.

4. de Sire A, Agostini F, Lippi L, Mangone M, Marchese S, Cisari C, Bernetti A, Invernizzi M. Oxygen-Ozone Therapy in the Rehabilitation Field: State of the Art on Mechanisms of Action, Safety and Effectiveness in Patients with Musculoskeletal Disorders. Biomolecules. 2021 Feb 26;11(3):356. doi: 10.3390/biom11030356.

5. Apuzzo D, Giotti C, Pasqualetti P, Ferrazza P, Soldati P, Zucco GM. An observational retrospective/horizontal study to compare oxygenozone therapy and/or global postural re-education in complicated chronic low back pain. Funct Neurol. 2014 Jan-Mar;29(1):31-9.

6. Rahimi-Movaghar V, Eslami V. The major efficient mechanisms of ozone therapy are obtained in intradiscal procedures. Pain Physician. 2012 NovDec;15(6):E1007-8.

7. Iliakis E, Valadakis V, Vynios DH, Tsiganos CP, Agapitos E. Rationalization of the activity of medical ozone on intervertebral disc: A Histological and Biochemical Study. Rivista di Neuroradiologia. 2001;14(1_suppl):23-30.

8. Simonetti L, Raffi L, Cenni P, Agati R, Leonardi M. Pharmacological Mechanisms Underlying OxygenOzone Therapy for Herniated Disc. Rivista di Neuroradiologia. 2003; 16(2_suppl_part2):201-204. 
9. Paoloni M, Di Sante L, Cacchio A, Apuzzo D, Marotta S, Razzano M, Franzini M, Santilli V. Intramuscular oxygen-ozone therapy in the treatment of acute back pain with lumbar disc herniation: a multicenter, randomized, double-blind, clinical trial of active and simulated lumbar paravertebral injection. Spine (Phila Pa 1976). 2009 Jun 1;34(13):1337-44. doi: 10.1097/BRS.0b013e3181a3c18d.

10. Biazzo A, Corriero AS, Confalonieri N. Intramuscular oxygen-ozone therapy in the treatment of low back pain. Acta Biomed. 2018 Mar 27;89(1):41-46. doi: 10.23750/abm.v89i1.5315.

11. Özcan Ç, Polat Ö, Çelik H, Uçar BY. The Effect of Paravertebral Ozone Injection in the Treatment of Low Back Pain. Pain Pract. 2019 Nov;19(8):821825. doi: 10.1111/papr.12812. Epub 2019 Jul 11.

12. Magalhaes FN, Dotta L, Sasse A, Teixera MJ, Fonoff ET. Ozone therapy as a treatment for low back pain secondary to herniated disc: a systematic review and meta-analysis of randomized controlled trials. Pain Physician. 2012 Mar-Apr;15(2):E11529.

13. Celestin J, Edwards RR, Jamison RN. Pretreatment psychosocial variables as predictors of outcomes following lumbar surgery and spinal cord stimulation: a systematic review and literature synthesis. Pain Med. 2009 May-Jun;10(4):639-53. doi: 10.1111/j.1526-4637.2009.00632.x.

14. Ware J Jr, Kosinski M, Keller SD. A 12-Item ShortForm Health Survey: construction of scales and preliminary tests of reliability and validity. Med Care. 1996 Mar;34(3):220-33. doi: 10.1097/00005650-199603000-00003.

15. Hinkle DE, Jurs SG, Wiersma W. Applied statistics for the behavioral sciences. Applied statistics for the behavioral sciences. 2nd ed. Boston: Houghton Mifflin Company. 1988.

16. Vowles KE, Gross RT, Sorrell JT. Predicting work status following interdisciplinary treatment for chronic pain. Eur J Pain. 2004 Aug;8(4):351-8. doi: 10.1016/j.ejpain.2003.10.009.

17. Corrêa JB, Costa LO, de Oliveira NT, Sluka KA, Liebano RE. Central sensitization and changes in conditioned pain modulation in people with chronic nonspecific low back pain: a case-control study. Exp Brain Res. 2015 Aug;233(8):2391-9. doi: 10.1007/s00221-015-4309-6. Epub 2015 May 12.

18. Mibu A, Nishigami T, Tanaka K, Manfuku M, Yono $\mathrm{S}$. Difference in the impact of central sensitization on pain-related symptoms between patients with chronic low back pain and knee osteoarthritis. J Pain
Res. 2019 May 29;12:1757-1765. doi: 10.2147/JPR.S200723.

19. Brosschot JF. Cognitive-emotional sensitization and somatic health complaints. Scand J Psychol. 2002 Apr;43(2):113-21. doi: 10.1111/1467-9450.00276.

20. Clark JR, Nijs J, Yeowell G, Holmes P, Goodwin PC. Trait Sensitivity, Anxiety, and Personality Are Predictive of Central Sensitization Symptoms in Patients with Chronic Low Back Pain. Pain Pract. 2019 Nov;19(8):800-810. doi: 10.1111/papr.12809. Epub 2019 Jul 10.

21. Cagnie B, Coppieters I, Denecker S, Six J, Danneels L, Meeus M. Central sensitization in fibromyalgia? A systematic review on structural and functional brain MRI. Semin Arthritis Rheum. 2014 Aug;44(1):68-75. doi: 10.1016/j.semarthrit.2014. 01.001. Epub 2014 Jan 8.

22. Tirelli U, Cirrito C, Pavanello M, Piasentin C, Lleshi A, Taibi R. Ozone therapy in 65 patients with fibromyalgia: an effective therapy. Eur Rev Med Pharmacol Sci. 2019 Feb;23(4):1786-1788. doi: 10.26355/eurrev_201902_17141.

23. Charney DS. Monoamine dysfunction and the pathophysiology and treatment of depression. J Clin Psychiatry. 1998;59 Suppl 14:11-4.

24. Wang JK. Antinociceptive effect of intrathecally administered serotonin. Anesthesiology. 1977; 47: 269-271.

25. Kranzler JD, Gendreau JF, Rao SG. The psychopharmacology of fibromyalgia: a drug development perspective. Psychopharmacol Bull. 2002 Winter;36(1):165-213.

26. Farhanchi A, Karkhanei B, Amani N, Aghajanloo M, Khanlarzadeh E, Emami Z. Association of Serum Serotonin and Pain in Patients with Chronic Low Back Pain before and after Spinal Surgery. Pain Res Treat. 2018 Sep 20;2018:4901242. doi: 10.1155/2018/4901242.

27. Moreno-Fernández A, Macías-García L, ValverdeMoreno R, Ortiz T, Fernández-Rodríguez A, MoliníEstrada A, De-Miguel M. Autohemotherapy with ozone as a possible effective treatment for Fibromyalgia. Acta Reumatol Port. 2019 Sep 29;44(3):244-249. English.

McGorry RW, Hsiang SM, Snook SH, Clancy EA, Young SL. Meteorological conditions and selfreport of low back pain. Spine (Phila Pa 1976). 1998 Oct 1;23(19):2096-102; discussion 2103. doi: 10.1097/00007632-199810010-00011.

Submitted: June 6, 2021 Accepted for publication: June 25, 2021 